\title{
Epigenetic and PPI Targeted Libraries from Life Chemicals ${ }^{+}$
}

\author{
Vasily Pinchuk *, George Bondar and Olga Balabon \\ Life Chemicals In., 1a DIXIE AVE, Niagara-on-the-Lake, ON LOS 1J0, Canada \\ * Correspondence: Vasily.Pinchuk@lifechemicals.com; Tel.: +1-250-448-6545 \\ + Presented at the 2nd Molecules Medicinal Chemistry Symposium (MMCS): Facing Novel Challenges in \\ Drug Discovery, Barcelona, Spain, 15-17 May 2019.
}

Published: 10 September 2019

Keywords: screening library; epigenetic; protein-protein interactions; drug discovery; virtual screening

Herein, we report our novel epigenetic and PPI screening libraries in response to a growing interest in these topics for discovery of novel drugs against emerging molecular targets.

Epigenetic inhibitors have been found to be very effective in cancer treatment. Epigenetic regulation of gene expression is not caused by changes in the nucleotide DNA sequences and implies modulation of chromatin structure (epigenetic marks). As epigenetic changes are dynamic, they can be reversed by epigenetic inhibitors. We designed three new Epigenetic Libraries using both structure- and ligand-based approaches. Virtual screening against various confirmed targets resulted in preparation of the Epigenetic Targeted Library and SIRT Targeted Library. Meanwhile, our Epigenetic Focused Library comprises compounds selected with 2D Fingerprint Similarity search.

Protein-protein interactions (PPIs) are involved in many important biological processes in life, so their regulation can be crucial for the treatment of numerous diseases. Low molecular weight PPI inhibitors able to selectively and potently modulate protein-protein interactions have recently reached clinical trials. Inspired by this promising breakthrough, we have prepared several PPI Focused Libraries of potential PPI inhibitors using various features within the ligand-based approach. A machine learning method (Decision Tree), known to be a useful tool to identify a PPI inhibitor profile, was used to predict which compounds could affect PPI. We also designed PPI Focused Libraries based on 2D Similarity Search (to Timbal DB or Binding DB, Pubmed DB). Alternatively, docking search against PDZ domain-containing proteins provided PPI Inhibitors Libraries.

(C) 2019 by the authors. Licensee MDPI, Basel, Switzerland. This article is an open access article distributed under the terms and conditions of the Creative Commons Attribution (CC BY) license (http://creativecommons.org/licenses/by/4.0/). 